\title{
Derechos laborales colectivos y democracia. Una discusión a partir del concepto voz horizontal de Guillermo O’Donnell*
}

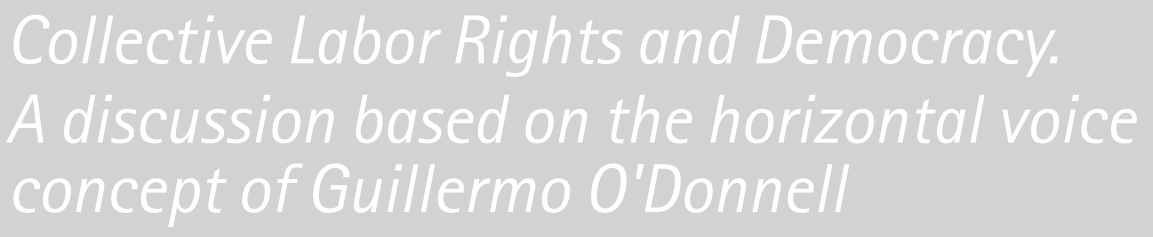

\section{Daniel Cerdas-Sandi"*}

\begin{abstract}
RESUMEN
Guillermo O'Donnell, en diálogo con la obra de Hirschman, señaló que las democracias, en su objetivo por consolidarse y mejorar su calidad, requerian de ciudadanos con voz. Sin embargo, no se refería sólo a una "voz vertical" en la que los ciudadanos alzan demandas a sus gobernantes, sino también una "voz horizontal" que permitiera la organización y la acción colectiva. A partir de esto, este artículo indaga en la legislación laboral como orden institucional que posibilita o restringe el acceso a voz horizontal por parte de grandes mayorias de la sociedad (los trabajadores). Mediante un diseño comparativo, se estudian dos casos de América Latina (Argentina y México), los cuales permiten comprender los vinculos entre derechos laborales colectivos, voz horizontal y democracia.
\end{abstract}

\section{PALABRAS CLAVE}

Guillermo O'Donnell, democracia, voz horizontal, voz vertical, derechos laborales colectivos.

\begin{abstract}
Guillermo O'Donnell, in dialogue with the work of Hirschman, pointed out that democracies, in their goal to consolidate and improve their quality, required citizens with a voice. However, it did not refer only to a "vertical voice" in which citizens raise demands on their rulers, but also a "horizontal voice" that would allow organization and collective action. From this, this article researches labor legislation as an institutional order that enables or restricts access to horizontal voice by large majorities of society (workers). Through a comparative design, two cases from Latin America (Argentina and Mexico) are studied, which allow to understand the links between collective labor rights, horizontal voice and democracy.
\end{abstract}

\section{PALABRAS CLAVE}

Guillermo O'Donnell, Democracy, Horizontal voice, Vertical voice, Collective labor rights

*Artículo recibido el 28 de julio de 2017 y aceptado para su publicación el 4 de diciembre de 2017.

** Universidad de Costa Rica y Consultor ort (danielcerdas.3@gmail.com) ORCID: 0000-0002-2554-3146 
SUM ARIO

1. Introducción

2. La relación entre democracia, voz horizontal y derechos laborales colectivos

3. Evidencia empírica desde la comparación: los casos de Argentina y México

4. Conclusiones

\section{INTRODUCCIÓN}

La democracia, como forma de organización política, ha experimentado cierto desprestigio en años recientes, en especial desde la crisis económica mundial de 2008. La ciudadanía percibe que sus intereses están por debajo de los intereses empresariales. Lo anterior recuerda la paradoja de Schmitter ${ }^{1}$ respecto a que la democracia pretende distribuir el poder relativamente igual, pero la economía capitalista distribuye bienes e ingresos relativamente desiguales. Es un hecho que la desigualdad ha tenido un aumento considerable, y se ha colocado como el gran desafío de la actualidad. ${ }^{2}$ Tal como demostró Przeworski, ${ }^{3}$ esto ha tenido negativos efectos sobre el funcionamiento de la democracia, en tanto "la desigualdad económica implica desigualdad política".

Estos dos fenómenos (negativa percepción de la democracia y desigualdad), tienen una relación directa con la forma en que se institucionalizan los derechos laborales colectivos. Por un lado, el aumento en la desigualdad, como dos estudios del Fondo Monetario Internacional (FMI) mostraron, está correlacionado con una caída en las tasas de sindicalización y cobertura de la negociación colectiva. Por otro lado se ha demostrado que para el buen funcionamiento de la democracia se requiere de una sociedad participativa. Esto se logra principalmente mediante una esfera laboral participativa; en ello, la libertad de sindicalizarse y negociar colectivamente es uno de los principales mecanismos democratizadores. ${ }^{5}$ En años recientes, también se ha comprobado la existencia de una estrecha relación entre la calidad de la democracia y el respeto de los derechos laborales colectivos. ${ }^{6}$

\footnotetext{
1 SCHMITTER, PHILIPPE, "Danger and Dilemmas of Democracy", Journal of Democracy, vol. 5, núm. 2, 1994, p. 66.

${ }^{2}$ StigltzZ, Joseph, El precio de la desigualdad, Bogotá, Taurus, 2012.

3 PrzeWorski, Adam, "Desigualdad económica, desigualdad política y redistribución", Conferencia Magistral 40 aniversario de Flacso-México, Ciudad de México, 28 de octubre de 2015.

${ }^{4}$ Francese, Maura y Mulas-Granados, Carlos, "Functional Income Distribution and Its Role in Explaining Inequality", ImF Working Paper, núm. 15/244, International Monetary Fund, 2015; y Jaumotte, Florence y Osorio Buitrón, Carolina, "Power from the People", Finance \& Development, vol. 52, núm. 1, pp. 29-31.

${ }^{5}$ Pateman, Carole, Participation and Democratic Theory, Cambridge, Cambridge University Press, 1970.

${ }^{6}$ Abounarb, Rodwan y Cingranelli, David, Human Rights and Structural Adjustment, Cambridge, Cambridge University Press, 2007.
} 
Como se puede ver, tales derechos juegan un importante rol en la consolidación de democracias de calidad mediante dos principales vías: reducen los efectos de la paradoja de Schmitter y fomentan la participación ciudadana. Asimismo, por la forma en que se configuran, pueden contribuir positivamente a dos aspectos fundamentales que ha tratado la ciencia política en los últimos años: rendición de cuentas (accountability, sobre todo vertical) y respuesta política a las demandas ciudadanas (resposiveness).

En ese sentido, este artículo discute como las características institucionales de los derechos laborales colectivos afectan (positiva o negativamente) la calidad de la democracia, en un sentido amplio (acceso de las mayorías a la arena política y a la toma de decisiones, y condiciones socioeconómicas de la población). Lo anterior a partir del concepto de voz horizontal propuesto por 0'Donnell. ${ }^{7}$ Para complementar la discusión teórica se muestran, mediante la comparación de dos casos (Argentina y México), los efectos y la forma en que se configuran estos derechos sobre la democracia de tals países durante el siglo XXI.

Metodológicamente, se hace uso de la estrategia comparativa conocida como diseño most similar: ${ }^{8}$ casos que presentan similitudes pero que en el fenómeno que se busca explicar difieren en sus resultados. Así, se han seleccionado dos casos con similitudes en el diseño institucional de los derechos laborales colectivos (en ambos casos se trata de modelos corporativos), que son democracias, al menos en un sentido minimalistas (eligen a sus representantes políticos mediante elecciones competitivas, los ganadores y perdedores reconocen los resultados y hay alternancia del poder). ${ }^{9}$ Asimismo, experimentaron reformas promercados y procesos de transición a la democracia, pero presentan resultados distintos en el ejercicio efectivo de los derechos laborales, con diferentes efectos sobre la democracia. Una acotación importante es que la selección de casos en este tipo de diseño most similar siempre se hace a partir de criterios relativos de semejanza. Tal como lo recuerda Lim, "debemos pensar el término 'más similar' como relativo”, pues es imposible encontrar países que tengan todas sus características iguales". ${ }^{10}$

\footnotetext{
${ }^{7}$ O'Donnell, Guillermo, "Sobre las fructíferas convergencias de las obras de Hirschman, salida, voz y lealtad y compromisos cambiantes: reflexiones a partir de la experiencia argentina reciente", en Guillermo O'Donnell (ed.), Contrapuntos. Ensayos escogidos sobre autoritarismo y democratización, Buenos Aires, Paidós, 1997, pp. 147-164.

${ }^{8}$ Przeworski, Adam y Teune, Henry, The Logic of Comparative Social Inquiry, Florida, Robert E. Krieger Publishing Company, 1982.

${ }_{9}^{9}$ Przeworski, Adam, "Minimalist Conception of Democracy: A Defense", en Robert Dahl, Alan lan Shapiro, y José Antônio Cheibub (eds.), The Democracy SourceBO0K, Massachusetts, The mit Press, 2003, pp.12-18.

${ }^{10}$ LIM, TIмотнY, Doing comparative politics: An introduction to approaches and issues, Boulder, Lynne Rienner Publishers, 2006.
} 
En vistas de esas limitaciones del diseño most similar, se recurre además a la técnica conocida como "comparación contextualizada", ${ }^{11}$ la cual permite determinar similitudes en lo que parece ser diferentes arreglos institucionales nacionales, ya que finalmente inducen a características similares de los derechos laborales colectivos. Al agrupar nuestra unidades de análisis, no precisamente tienen la misma institucionalidad laboral, pero sí es posible considerarlos semejantes en la medida en que se pueden identificar "puntos de fricción", que son, a su vez, las fuentes potenciales de conflicto entre representantes laborales y empresariales. ${ }^{12}$

\section{LA RELACIÓN ENTRE DEMOCRACIA, VOZ HORIZONTAL Y DERECHOS LABORALES COLECTIVOS}

La definición mínima de democracia incluye la libertad de asociación como uno de sus pilares. ${ }^{13}$ Por lo general, y en especial con el auge del modelo neoliberal, esta libertad se ha reducido a la participación en los procesos electorales (desde su valoración desde la academia, hasta las políticas públicas). Con ello, se ha dejado de lado el rol determinante de las organizaciones de la sociedad civil en el mejoramiento y consolidación de la democracia, en tanto estructuras modernas de representación de los intereses.

El reconocimiento de la libertad de asociación en un sentido amplio, más allá de lo electoral, se presenta como uno de los grandes retos para lograr avances en la calidad de la democracia. Los partidos políticos, aunque necesarios para el buen funcionamiento de un régimen democrático, presentan muchas limitaciones para la representación de los intereses de la sociedad. Con ello, las organizaciones secundarias son los verdaderos sujetos políticos, que crean y estimulan la pluralidad y tolerancia política, fomentan el civismo y la educación política, colaboran en la legitimación del orden democrático (verificación del ejercicio del poder), promueven la deliberación pública y suscitan la gobernanza. ${ }^{14}$

\footnotetext{
"Locke, Richard y Thelen, Kathleen, "Apples and Oranges Revisited: Contextualized Comparison and the Study of Comparative Labor Studies", Politics \& Society, vol. 23, núm. 3, pp. 337-367.

${ }^{12}$ Bensusán, Graciela, "Organizing Workers in Argentina, Brazil, Chile and Mexico: The Authoritarian-Corporatist Legacy and Old Institutional Designs in a New Context", Theoretical Inquiries in Law, vol. 17, núm. 1, pp. 131-161.

${ }^{13}$ Przeworski, Adam, "Minimalist Conception of Democracy: A Defense", en Robert Dahl, Alan lan Shapiro, y José Antônio Cheibub (eds.), The Democracy SourceB0OK, Massachusetts, The mit Press, 2003, pp. 12-18.

${ }^{14}$ Fung, Archon, "Associations and democracy: Between theories, hopes, and realities", Annual review of sociology, vol. 29, núm. 1, pp. 515-539.
} 
Específicamente en relación con las organizaciones laborales y sus efectos sobre la democracia, hay importantes aportes desde las ciencias jurídicas. Gordon ${ }^{15}$ introdujo el concepto de ciudadanía laboral, que se refiere a la condición de ser miembro de una organización de trabajadores y al acto de participación en los procesos de toma de decisiones de la organización. El esfuerzo por construir la ciudadanía conllevaría ejercer el poder en el lugar de trabajo, la comunidad y la arena política, con el fin de lograr el reconocimiento político-social y mejorar sus vidas.

También desde el derecho se ha estudio cómo los sindicatos han sido clave, en regímenes autoritarios, para la creación de condiciones para que la democracia prospere. ${ }^{16}$ Otros estudios ${ }^{17}$ han argumentado que la existencia de instituciones democráticas en el lugar de trabajo podría contribuir a que los trabajadores desarrollen capacidades para participar en el sistema político. Asimismo, se ha llegado a establecer que hay una función de empoderamiento asociada con las normas del derecho laboral que fortalece la libertad positiva, que se traduce en una mejor canalización de los intereses ciudadanos. ${ }^{18}$

Estos aportes han logrado mostrar las relaciones entre la institucionalidad laboral y la democracia, han estado especialmente centradas en las repercusiones que ese orden tiene sobre lo que O'Donnell, a partir de la obra de Albert Hirschman, ${ }^{19}$ llamó voz vertical. ${ }^{20}$ Es decir, la voz o las demandas que los ciudadanos, trabajadores, clientes, entre otros, elevan hacia "arriba", donde se encuentran las instancias de poder y tienen la capacidad de resolver las solicitudes.

El ejercicio de voz vertical guarda relación con los conceptos de accountability y de responsiveness. En el primer caso, la voz vertical puede contribuir a la transparencia del ejercicio del poder mediante mecanismos diversos de control ciudadano que permitan medidas directas de rendición de cuentas a la ciudadanía.

\footnotetext{
${ }^{15}$ Gordon, Jennifer, "Transnational Labor Citizenship", Southern California Law Review, vol. 80, 2007, 503-587.

${ }^{16}$ Fick, BarbarA, "Not Just Collective Bargaining: The Role of Trade Unions in Creating and Maintaining a Democratic Society", WorkingUSA: The Journal of Labor and Society, vol. 12, núm. 2, 2009, pp. 249-264.

${ }^{17}$ Kolben, KeVIN, "Labour Regulation, Human Capacities and Industrial Citizenship", en Shelley Marshall y Colin Fenwick (eds.), Labour Regulation and Development, Cheltenham - Geneva, Edward Elgar Publishing - IL0, 2016, pp. 60-81.

${ }^{18}$ DeAkIN, SImon, "Labor Law and Development in the Long Run", en Shelley Marshall y Colin Fenwick (eds.), Labour Regulation and Development, Cheltenham - Geneva, Edward Elgar Publishing - IL0, 2016, pp. 33-59.

${ }^{19}$ Hirschman, Albert, Exit, Voice and Loyalty: Responses to Decline in Firms, Organizations and States, Cambridge, Harvard University Press, 1970.

${ }^{20}$ O'Donnell, Guillermo, "Sobre las fructiferas convergencias de las obras de Hirschman, salida, voz y lealtad y compromisos cambiantes: reflexiones a partir de la experiencia argentina reciente", en Guillermo O'Donnell (ed.), Contrapuntos. Ensayos escogidos sobre autoritarismo y democratización, Buenos Aires, Paidós, 1997, pp. 147-164. El presente artículo se enfoca en el concepto de voz vertical en el sentido colectivo del término. Existe también la posibilidad de que se dé un ejercicio individual del mismo (por ejemplo, poner una queja en una oficina estatal, solicitar información a una dependencia gubernamental, entre otras).
} 
La voz vertical sería un elemento clave en la posibilidad de que los ciudadanos cuestionen la labor de los tomadores de decisiones públicas y recibir respuestas sobre esos cuestionamientos.

Por otra parte, un efectivo ejercicio de la voz vertical es esencial para que los gobernantes obren según los intereses de la ciudadanía. Como señaló Sánchez y Sánchez, "una democracia será de mayor calidad en la medida en que los ciudadanos ejerzan plenamente sus derechos y obligaciones y puedan también exigir a sus gobernantes que tomen decisiones acorde a sus preocupaciones políticas urgentes, a sus preferencias y necesidades". ${ }^{21}$

Sin embargo, es 0'Donnell quien llama la atención sobre otro tipo de voz: la voz horizontal. Dicha voz no está establecida de manera paralela a la vertical, ni se trata de una derivación de esta; más bien es una condición necesaria para que pueda darse la voz vertical. Sin voz horizontal, difícilmente pueda darse la voz vertical. La voz horizontal se refiere a la institucionalidad que permite $o$ restringe la acción colectiva de los ciudadanos frente a quienes tienen poder. Es la forma en que un determinado entramado de normas y prácticas se establece para fomentar-facilitar o bien restringir-impedir la organización de quienes, en las relaciones de poder, son considerados la "parte débil". De esta forma, la voz horizontal se presenta como un elemento pilar en las democracias, pues en última instancia sobre ella descansa una serie de características propias de un buen régimen democrático. Es muy probable que un sistema político que establezca una institucionalidad restringida para el efectivo ejercicio de la voz horizontal (en sus distintas expresiones) presente muchas deficiencias en el funcionamiento de sus instituciones democráticas.

La voz horizontal permite el reconocimiento de los intereses colectivos en común y, a partir de ahí, la organización de la acción colectiva. Así, funciona como un mecanismo para la formación de la identidad colectiva, el autorreconocimiento de un "nosotros". Con ella se llega a notar que de forma individual se está en desventaja frente a quienes están en el poder, pero que a través de la organización colectiva se logra la reducción de esas asimetrías de poder. Asimismo, le da a una constelación de individuos la posibilidad de ser escuchados por los de "arriba”, pero más importante aún, que sus demandas sean cumplidas. Da la posibilidad de ejercer de forma efectiva la voz vertical colectiva. Esto se pueda dar ya sea mediante formas directas (protestas, huelgas, plantones, entre

\footnotetext{
${ }^{21}$ Sánchez y Sánchez, Carlos Luis, "Calidad de la democracia y respuesta política. Responsiveness", Apuntes Electorales, año 15, núm. 54, pp. 147-150.
} 
otros), o de manera indirecta, cuando se eligen individuos para hablar ante las autoridades en representación de los intereses del colectivo. ${ }^{22}$

En este sentido, 0’Donnell agregó:

La posibilidad de hacer uso de la voz horizontal sin restricciones o peligros serios es un rasgo constitutivo de un contexto democrático (y, en general, no represivo). La voz horizontal es una condición necesaria de la existencia de una voz vertical colectiva razonablemente autónoma respectos de "los de arriba”. Ésta, a su vez, es una condición necesaria de la existencia de un contexto democrático. ${ }^{23}$

Un espacio determinante para evaluar las condiciones de la voz horizontal, en países constituidos bajo modelos económicos capitalistas, es la institucionalidad laboral relativa a los derechos laborales colectivos. Este aspecto también fue analizado por 0’Donnell, ${ }^{24}$ quien apuntó primero que las luchas de los trabajadores por ser reconocidos por el Estado y la burguesía como un actor colectivo conllevaron la expansión de los derechos democráticos en la esfera de la ciudadanía política, en las relaciones laborales y con el Estado. En esto los sindicatos y los partidos de base laboral tuvieron un rol protagónico; impulsaron en el largo plazo constantes luchas para lograr avances en esos derechos.

En los países industrializados, y en algunos casos de América Latina, el Estado y la burguesía terminaron por aceptar "la representatividad colectiva de las organizaciones obreras y ha tenido que negociar con ellas la expansión de un amplio conjunto de derechos. En contraste en una relación social arcaica no hay reconocimiento de los actores colectivos". 25

La institucionalidad de los derechos laborales colectivos, en especial -para el caso de América Latina- la que refiere al derecho a organizarse de manera autónoma en sindicatos, al derecho a negociar colectivamente las condiciones de trabajo y al derecho a la huelga, es la que tiene mayor impacto en la democratización, no sólo de las relaciones laborales, sino también de la sociedad en

\footnotetext{
${ }^{22}$ O'Donnell, Guillermo, "Sobre las fructíferas convergencias de las obras de Hirschman, salida, voz y lealtad y compromisos cambiantes: reflexiones a partir de la experiencia argentina reciente", en Guillermo 0'Donnell (ed.), Contrapuntos. Ensayos escogidos sobre autoritarismo y democratización, Buenos Aires, Paidós, 1997, p. 150.

${ }^{23}$ O'Donnell, Guillermo, "Sobre las fructíferas convergencias de las obras de Hirschman, salida, voz y lealtad y compromisos cambiantes: reflexiones a partir de la experiencia argentina reciente", en Guillermo 0'Donnell (ed.), Contrapuntos. Ensayos escogidos sobre autoritarismo y democratización, Buenos Aires, Paidós, 1997, p. 150.

${ }^{24}$ O'Donnell, Guillermo, "Transiciones, Continuidades y Algunas Paradojas", en Guillermo O'Donnell (ed.), Contrapuntos. Ensayos escogidos sobre autoritarismo y democratización, Buenos Aires, Paidós, 1997, pp. 246-247.

${ }^{25}$ O'Donnell, Guillermo, "Transiciones, Continuidades y Algunas Paradojas", en Guillermo O'Donnell (ed.), Contrapuntos. Ensayos escogidos sobre autoritarismo y democratización, Buenos Aires, Paidós, 1997, p. 247.
} 
general. En contextos en que amplias masas de la sociedad tiene acceso a ejercer estos derechos se les permite una mejor incorporación a la arena política, ${ }^{26}$ y con ello mayores posibilidades de incidencia en las políticas públicas, acorde con sus intereses.

En primer lugar, la posibilidad de organizarse libremente en sindicatos autónomos permite establecer una institucionalidad que garantiza el desarrollo de la voz horizontal para los trabajadores. A través del sindicato, los trabajadores reducen las asimetrías de poder frente a sus empleadores y frente al Estado. No se trata ya de una relación donde el asalariado, de forma individual, eleva sus demandas a su empleador o a las autoridades políticas, sino de un colectivo.

En segundo lugar, el derecho a la negoción colectiva brinda otros elementos de fortalecimiento de la voz horizontal. Las condiciones de la relación salarial no se negocian de forma individual, sino de manera colectiva, reduciendo a su vez las asimetrías de poder. Además, mediante el contrato colectivo se evita la fragmentación de los intereses de los trabajadores y sus recursos de poder, a la vez que permite el reconocimiento de un "nosotros" que tiene intereses comunes.

Finalmente, el derecho al ejercicio de la huelga juega un rol determinante como recurso de poder. Es el principal instrumento de presión de los trabajadores frente al empleador y al Estado. Es la herramienta última para hacer valer su voz vertical colectiva. Su efectivo ejercicio depende en buena parte de la forma institucional en que se establece el derecho a la sindicalización y la negociación colectiva. Así, sindicalización, negociación colectiva y huelga se establecen como una tríada central para el desarrollo de la voz horizontal y del efectivo ejercicio de una voz vertical colectiva.

Aunque se ha señalado la importancia de la organización colectiva de los intereses de los trabajadores como forma de voz horizontal que permite una voz vertical colectiva, esto no implica la renuncia de la autonomía e independencia de cada trabajador como individuo. Más bien no se ensalza una especie de colectivismo como organización ideal de los trabajadores y ciudadanos. Una adecuada voz horizontal, en contextos democráticos, permitiría que cada quien se organice a partir de lo que considera su propio beneficio, lo que lleva al reconocimiento de intereses comunes con sus semejantes. La voz horizontal no elimina las individualidades, ni somete a las "partes" al criterio del "todo", solamente garantiza la reducción de las asimetrías de poder político y económico a las que se enfrenta el ciudadano de manera individual en las democracias liberales capitalistas.

\footnotetext{
${ }^{26}$ Coller, Ruth Berins y Coller, David, Shaping the Political Arena. Critical Junctures, The Labor Movement, and Regime Dynamics in Latin America, Notre Dame, University of Notre Dame Press, 2002.
} 


\section{Evidencia empírica desde la comparación: los casos de Argentina y MÉXico}

Para contrastar con la realidad estos aspectos teóricos, en este apartado se analizan las características que han presentado los derechos laborales colectivos durante el siglo XXI en Argentina y México, y los efectos que esto ha tenido sobre la democracia. Es decir, no se hace una evaluación limitada a lo electoral, pues "la democracia no sólo implica ciertos procedimientos para seleccionar y controlar el poder político, sino también objetivos y resultados que se pretenden alcanzar, tales como el desarrollo económico, la justicia social o la igualdad". ${ }^{27}$

Tanto en Argentina como en México, los regímenes laborales comparten legados de corporativismo estatal. Entre ellos destacan el control estatal sobre las relaciones laborales, importantes subsidios financieros y apoyos institucionales a los sindicatos y una estructura de la organización sindical que restringe la competencia. ${ }^{28}$ Sin embargo, aun cuando ambos casos tienen origen en los procesos de incorporación de los trabajadores a la arena política, a través de partidos políticos hegemónicos, durante el periodo de industrialización por sustitución de importaciones y bajo una estrategia de desarrollo guiado por el Estado, ${ }^{29}$ a partir de la década de 1980, con los cambios económicos (neoliberalismo) y políticos (democratización), estos modelos laborales han tomado caminos divergentes, aun cuando sus características de corporativismo se han mantenido. ${ }^{30}$

Estas divergencias en las trayectorias de los modelos laborales son el resultado de la manera en que se dio la doble transición (económica y política). La secuencia en que se dieron estos procesos influyó en las capacidades de los sindicatos al momento de acceder a recursos institucionales y en el aprovechamiento de las alianzas partidistas que mantenían. ${ }^{31}$ Así, cuando se dio la transición primero democrática y después económica, como en el caso argentino, los sindicatos se fortalecieran para enfrentar las reformas económicas y la

\footnotetext{
${ }^{27}$ BarReda, Maiket, "La calidad de la democracia: Un análisis comparado de América Latina", Política y gobierno, vol. 18, núm. 2, p. 268.

${ }_{28}$ Bensusán, Graciela y Cook, Maria Lorena, "State-corporatist Legacies and Divergent Paths", en Stephen Crowley, Maria Lorena Cook (eds.), Working through the Past: Labor and Authoritarian Legacies in Comparative Perspectives, Ithaca, Cornell University Press, 2015, p. 143-144.

${ }^{29}$ Collier, Ruth Berins y Collier, David, Shaping the Political Arena. Critical Junctures, The Labor Movement, and Regime Dynamics in Latin America, Notre Dame, University of Notre Dame Press, 2002.

${ }^{30}$ Cook, Maria Lorena, The Politics of Labor Reform in Latin America: Between Flexibility and Rights, University Park, The Pennsylvania State University Press, 2007; Bensusán, Graciela y Cook, Maria Lorena, "Political Transition and Labor Revitalization in Mexico", en Daniel Cornfield y Holly McCammon (eds.), Labor Revitalization: Global Perspectives and New Initiatives, Vanderbilt, Elsevier, 2003, pp. 229-267.

${ }^{31}$ Cook, Maria Lorena, "Labor Reform and Dual Transitions in Brazil and the Southern Core", Latin American Politics and Society, vol. 44, núm. 1, pp. 1-34.
} 
flexibilidad laboral, disminuyendo así los efectos negativos que éstas pudieron tener sobre los derechos colectivos. Cuando se dio de manera inversa, como en el caso mexicano, las reformas económicas fueron acompañadas de agresivos cambios laborales (no tanto en términos de reformas a las normas, sino más bien en la efectividad de su aplicación) que flexibilizaron profundamente las relaciones laborales. Fue especialmente notorio en la caída de los salarios y en las restricciones a la negociación colectiva. ${ }^{32}$

Estas características de los procesos de transición permitieron en el caso de Argentina -a pesar de los embates contra los derechos laborales colectivos experimentados durante la década de 1990-, que se mantuvieran importantes recursos poder para los trabajadores. De esta forma, a partir del año 2003, ante una nueva coyuntura política, se recuperan muchas de las instituciones laborales que beneficiaban a los trabajadores, ya fuera mediante la reactivación institucional o por medio de nueva legislación. Esto ha propiciado una revitalización del movimiento sindical. ${ }^{33}$

En contraste, en México la legislación sobre los derechos laborales colectivos, aunque inicialmente otorgaba importantes recursos de poder a los trabajadores (lo que daba garantía de acceder a algún tipo de voz horizontal), con el giro neoliberal del modelo económico se desactivaron instituciones laborales colectivas. Lo anterior restringió el acceso a la voz horizontal. Por ejemplo, el Consejo de Salarios Mínimos dejó de funcionar como órgano que velaba por ingresos salariales dignos para los trabajadores. Otras instituciones tripartitas pasaron a responder únicamente a los intereses empresariales y del Poder Ejecutivo. Todo esto fue posible en tanto se mantuvieron intactos los controles sobre la estructura de la organización sindical, lo cual permitió contar con cúpulas sindicales que no responden a los intereses de los trabajadores. Esto, a su vez, llevó a facilitar la implementación de serias restricciones en los derechos colectivos. $^{34}$

Esto ha persistió aun después de la reforma laboral de 2012, proceso que reveló una vez más el carácter del modelo laboral mexicano de las últimas décadas. Tanto empleadores y como la dirigencia sindical tradicional, a través de sus vínculos en el Poder Ejecutivo y Legislativo, se opusieron a cualquier

\footnotetext{
32 Bensusán, Graciela y Cook, Maria Lorena, "State-corporatist Legacies and Divergent Paths", en Stephen Crowley, Maria Lorena Cook (eds.), Working through the Past: Labor and Authoritarian Legacies in Comparative Perspectives, Ithaca, Cornell University Press, 2015, pp. 152-153.

${ }^{33}$ EtChemendy, Sebastián y Coller, Ruth Berins, "Down but not out: Union resurgence and segmented neocorporatism in Argentina (2003-2007)", Politics \& Society, vol. 35, núm. 3, pp. 363-401.

${ }^{34}$ Bensusán, Graciela, El modelo mexicano de regulación laboral, México, Flacso, 2000.
} 
cambio que representara avances democratizadores en la estructura de representación de los trabajadores y garantizaron así la continuidad de las restricciones sobre el ejercicio de los derechos laborales colectivos. ${ }^{35}$

A partir de estas diferencias entre estos modelos laborales corporativistas, se han llegado a clasificar como "corporativismo fuerte" el argentino y "corporativismo rígido" el mexicano. ${ }^{36} \mathrm{El}$ modelo argentino ha logrado constituirse como una estructura que da importantes recursos de poder a los trabajadores, incluso cuando han experimentado periodos adversos. La fuerza emanada de la institucionalidad laboral ha permitido la recuperación del sindicalismo como actor social y político relevante. En el caso mexicano, la institucionalidad laboral impide la democratización de las estructurales sindicales, y mantiene una dirigencia que responde en el nivel macro al Gobierno (espacios de diálogo social o en el Congreso) y en el nivel micro a los empleadores. Esta estructura rígida, al mismo tiempo, permite la flexibilidad de las condiciones de trabajo, pues los patrones cuentan con el aval y respaldo sindical para violar las protecciones laborales. ${ }^{37}$ En la tabla 1 se muestran las principales características institucionales de ambos modelos laborales.

\footnotetext{
${ }^{35}$ Bensusán, Graciela y Middlebrook, KeVIn J., Sindicatos y política en México: cambios, continuidades y contradicciones, Flacso - UAM - Clacso, 2013.

${ }^{36}$ Bensusán, Graciela, "Organizing Workers in Argentina, Brazil, Chile and Mexico: The Authoritarian-Corporatist Legacy and Old Institutional Designs in a New Context", Theoretical Inquiries in Law, vol. 17, núm. 1, pp. 131-161. ${ }_{37}$ Bensusán, Graciela, "Organizing Workers in Argentina, Brazil, Chile and Mexico: The Authoritarian-Corporatist Legacy and Old Institutional Designs in a New Context", Theoretical Inquiries in Law, vol. 17, núm. 1, pp. 131-161.
} 
Tabla 1. Principales características de las instituciones laborales colectivas en Argentina y México

\begin{tabular}{|c|c|c|c|}
\hline \multirow{2}{*}{\multicolumn{2}{|c|}{ Ratificación de convenios oIT }} & Argentina & México \\
\hline & & 87 y 98 & 87 \\
\hline \multicolumn{2}{|c|}{ Aspectos críticos } & $\begin{array}{l}\text { Restricciones a } \\
\text { los sindicatos con } \\
\text { reconocimiento pero sin } \\
\text { "personería gremial" }\end{array}$ & $\begin{array}{l}\text { Opción discrecional para } \\
\text { seleccionar contraparte } \\
\text { sindical en la negociación } \\
\text { colectiva por parte del } \\
\text { empleador }\end{array}$ \\
\hline \multicolumn{2}{|c|}{ Unidad sindical vs. pluralismo } & $\begin{array}{l}\text { Membresía obligatoria a } \\
\text { nivel sectorial (industria) }\end{array}$ & $\begin{array}{l}\text { Formalmente el pluralismo, } \\
\text { pero con el monopolio de la } \\
\text { representación a través de } \\
\text { closed-shop }\end{array}$ \\
\hline \multicolumn{2}{|c|}{ Autoridad de registro sindical } & $\begin{array}{l}\text { Ministerio de Trabajo. Se } \\
\text { reconoce el monopolio de } \\
\text { la negociación colectiva } \\
\text { para el sindicato más } \\
\text { representativo }\end{array}$ & $\begin{array}{l}\text { Ministerio de Trabajo } \\
\text { (jurisdicción federal) o Juntas } \\
\text { de Conciliación y Arbitraje } \\
\text { (Jurisdicción local) }\end{array}$ \\
\hline \multicolumn{2}{|c|}{$\begin{array}{l}\text { Protección contra las prácticas } \\
\text { antisindicales }\end{array}$} & Sí & No \\
\hline \multicolumn{2}{|c|}{ Negociación colectiva } & $\begin{array}{l}\text { Centralizado a nivel } \\
\text { industrial o sectorial; y } \\
\text { empresa }\end{array}$ & $\begin{array}{l}\text { Descentralizado a nivel de } \\
\text { empresa y pocos contratos de } \\
\text { ley (industria o sector) }\end{array}$ \\
\hline \multicolumn{2}{|c|}{$\begin{array}{l}\text { Resolución de disputas por el } \\
\text { Estado }\end{array}$} & st & $\begin{array}{l}\text { Sólo si ambas partes están de } \\
\text { acuerdo }\end{array}$ \\
\hline \multicolumn{2}{|c|}{ Alcance del derecho de huelga } & $\begin{array}{l}\text { Amplio, arbitraje a } \\
\text { petición de una de las } \\
\text { partes }\end{array}$ & $\begin{array}{l}\text { Amplio en la legislación, casi } \\
\text { imposible de ejercer en la } \\
\text { práctica }\end{array}$ \\
\hline \multirow{2}{*}{$\begin{array}{l}\text { Recursos } \\
\text { sindicales }\end{array}$} & $\begin{array}{l}\text { Efectos de la } \\
\text { negociación } \\
\text { colectiva }\end{array}$ & $\begin{array}{l}\text { Erga omnes. Los no } \\
\text { miembros pueden ser } \\
\text { obligados a pagar cuotas } \\
\text { de afiliación al sindicato } \\
\text { con mantenimiento } \\
\text { del monopolio de } \\
\text { representación }\end{array}$ & Erga omnes \\
\hline & $\begin{array}{l}\text { Representación } \\
\text { sindical en } \\
\text { el lugar de } \\
\text { trabajo (fuerte } \\
\text { recurso para la } \\
\text { sindicalización) }\end{array}$ & $\begin{array}{l}\text { Por ley en lugares con } \\
\text { diez o más trabajadores, y } \\
\text { por negociación colectiva }\end{array}$ & $\begin{array}{l}\text { Excepcional por ley y } \\
\text { negociación colectiva }\end{array}$ \\
\hline
\end{tabular}




\begin{tabular}{|c|c|c|}
\hline Fuentes de financiamiento & $\begin{array}{l}\text { Cuotas de afiliación } \\
\text { sindical y las cotizaciones } \\
\text { patronales estipuladas en } \\
\text { los convenios colectivos. } \\
\text { Los sindicatos administran } \\
\text { los recursos de servicios } \\
\text { de seguridad social } \\
\text { (pensión y salud) para } \\
\text { todos los trabajadores } \\
\text { asalariados }\end{array}$ & $\begin{array}{l}\text { Cuotas de afiliación sindical } \\
\text { obligatorias y contribuciones } \\
\text { patronales estipuladas en } \\
\text { convenios colectivos }\end{array}$ \\
\hline $\begin{array}{l}\text { Afiliación e Incentivos para } \\
\text { reclutar miembros }\end{array}$ & $\begin{array}{l}\text { Voluntario; los sindicatos } \\
\text { prestan una variedad de } \\
\text { servicios: salud, pensiones, } \\
\text { turismo, deportes, } \\
\text { asesoramiento jurídico y } \\
\text { otros }\end{array}$ & $\begin{array}{l}\text { Obligatoria y negativa (closed } \\
\text { shop) }\end{array}$ \\
\hline
\end{tabular}

Fuente: BensusÁn, Graciela, “Organizing Workers in Argentina, Brazil, Chile and Mexico: The Authoritarian-Corporatist Legacy and Old Institutional Designs in a New Context", Theoretical Inquiries in Law, vol. 17, núm. 1, pp. 158-160 [la traducción es nuestra].

Estas caracteristicas que han asumido los derechos laborales colectivos en Argentina, en especial a partir de 2003, han permitido la revitalización de la organización sindical. Se han convertido en un importante actor político-social, con importantes impactos en la democratización del país, aun cuando persisten rezagos. Las primeras medidas a favor de los derechos laborales colectivos se hicieron mediante la reactivación de los espacios de diálogo social desactivados durante el periodo neoliberal.

El Gobierno de Kirchner, en 2003, convocó el Consejo del Empleo, la Productividad y el Salario Mínimo, Vital y Móvil, ${ }^{38}$ desactivado desde mediados de la década de 1990. En el Consejo, la representación gubernamental adoptó posiciones pro-sindicales, lo que se tradujo en una activa política salarial del Estado y un aumento progresivo de los salarios. ${ }^{39}$ Esto representó un cambio de paradigma, pues las posturas que habían insistido en señalar la falta de flexibilización laboral como uno de los principales problemas de la economía perdieron terreno. ${ }^{40} \mathrm{~A}$ estos cambios le siguió la aprobación de una nueva le-

\footnotetext{
${ }^{38}$ Por lo general se le conoce sólo como Consejo de Salario Mínimo.

${ }^{39}$ Senén González, Cecilia, "La revitalización sindical en Argentina durante los Kirchner", Revista Trabajo, vol. 5, núm. 8, pp. 46-47.

40 Novick, Marta y Tomada, Carlos, "Argentina 2003-2006: Crecimiento Económico con Empleo Decente ¿Un Nuevo Modelo para América Latina?", en Marta Novick, Carlos Tomada, Mario Damill, Roberto Frenkel y Roxana Maurizio Ginebra (eds.), Tras la crisis: el nuevo rumbo de la política económica y laboral en Argentina y su impacto, Instituto
} 
gislación ${ }^{41}$ para recuperar varias de las normas perdidas durante el periodo neoliberal.

Estos cambios, vía reactivación institucional y nueva legislación, no se dieron de manera inercial con la llegada de gobierno de izquierda, sino que las huelgas y la movilización social, es decir, la acción colectiva de los trabajadores, fue necesaria para impulsar los avances protrabajadores. ${ }^{42} \mathrm{Al}$ mismo tiempo, se experimentó una ampliación en la tasa de sindicalización (fue de $31.7 \%$ en $2000,37 \%$ en 2005 , y $39.7 \%$ en 2006) y en la tasa de cobertura de la negociación colectiva (el porcentaje de cobertura de la negociación colectiva sobre el empleo asalariado total era de $13.30 \%$ en 2004 y llegó a 36.5\% en 2010; la cifra llega a 87.5\% si sólo se considera el trabajo asalariado registrado del sector privado).$^{43}$ El ejercicio de la voz horizontal jugó un rol determinante en los avances. Precisamente se logró reducir el trabajo informal no agrícola: $43.1 \%$ en $2001,37.1 \%$ en 2008 , y $33.1 \%$ en $2015 ;{ }^{44}$ reducir la tasa de desempleo: de $17.3 \%$ en 2003 a 7.9\% en 2008, y 6.5\% en 2015; y se dio un aumento real en 300\% del salario mínimo entre 2000 y $2010 . .^{45}$

Todos estos cambios en el ejercicio y alcances de los derechos laborales colectivos tuvieron impactos distributivos a favor de los trabajadores. La participación de los salarios en el PIB mantuvo un comportamiento ascendente a partir del 2004 (véase gráfico 1), mientras que la desigualdad mostró una disminución sostenida de 2003 a 2011 (véase gráfico 2). La orT llegó a señalar

Internacional de Estudios Laborales, 2007.

${ }^{41}$ En 2004 se aprobó un nueva ley laboral (Ley de Ordenamiento Laboral 25.877) que constituiría uno de los mayores avances a favor del PAT, pues se recuperaba la noción de ultraactividad en la negociación colectiva. Además, la ley fomentaba de nuevo la negociación colectiva sectorial.

${ }^{42}$ Senén Gonzalezz, Cecilla y Del Bono, Andrea, "Introducción", en Cecilia Senén González y Andrea del Bono, La revitalización sindical en Argentina: alcances y perspectivas, San Justo, Prometeo - Universidad Nacional de la Matanza, 2013, pp. 7-29.

${ }^{43}$ La información sobre la tasa de sindicalización y la tasa de cobertura de la negociación colectiva y homologación de acuerdos y convenios colectivos provienen de Senén González, Cecilia, Trajtemberg, David y Medwid, BÁrbara, "La negociación colectiva en la Argentina 1991-2007", en Cecilia Senén González y Patricia Schettini (comps.), Trabajo y relaciones laborales en Argentina: sector formal e informal, Buenos Aires, Universidad Nacional de La Matanza - Prometeo, 2009, pp. 117-139; SenÉn González, CeclliA, "La revitalización sindical en Argentina durante los Kirchner", Revista Trabajo, vol. 5, núm. 8, pp. 52-54; MARTICORENA, ClarA, "Revitalización sindical y negociación colectiva en Argentina (2003-2011)", Perfiles latinoamericanos, vol. 23, núm. 46, p. 183; Bensusán, Graciela y Cook, Maria Lorena, "State-corporatist Legacies and Divergent Paths", en Stephen Crowley, Maria Lorena Cook (eds.), Working through the Past: Labor and Authoritarian Legacies in Comparative Perspectives, Ithaca, Cornell University Press, 2015, p. 157.

${ }_{44}$ Los datos sobre la evolución de la tasa de informalidad provienen de oIr, Informe Mundial Sobre Salarios 2014/2015. Salarios y Desigualdad de Ingresos, Ginebra, olt, 2015; y olt, Informe Mundial Sobre Salarios 2014/2015. Salarios y Desigualdad de Ingresos, Ginebra, oІт, 2015.

${ }^{45}$ Los datos sobre la evolución del salario mínimo real y sobre desempleo provienen de dos fuentes: la base de datos de la Cepal y oır, Panorama Laboral 2011: América Latina y el Caribe, Lima, oІт, 2011, p. 46. 
que Argentina presentaba la mayor disminución de la desigualdad durante la primera década del siglo xxI en todo el mundo; pero además destacaba que el efecto del desempeño del mercado laboral determinó 87\%. ${ }^{46}$

Estas estadísticas de Argentina no pueden interpretarse limitadamente como cambios socioeconómicos. Representaron importantes avances en términos de ciudadanía y son indicador clave al momento de evaluar los regímenes democráticos (tantos en aspectos relativos al contenido como al resultado, según la conceptualización desarrollada por Morlino). ${ }^{47}$

Gráfico 1. Participación de los salarios en el producto interno bruto en Argentina y México, 2000-2011

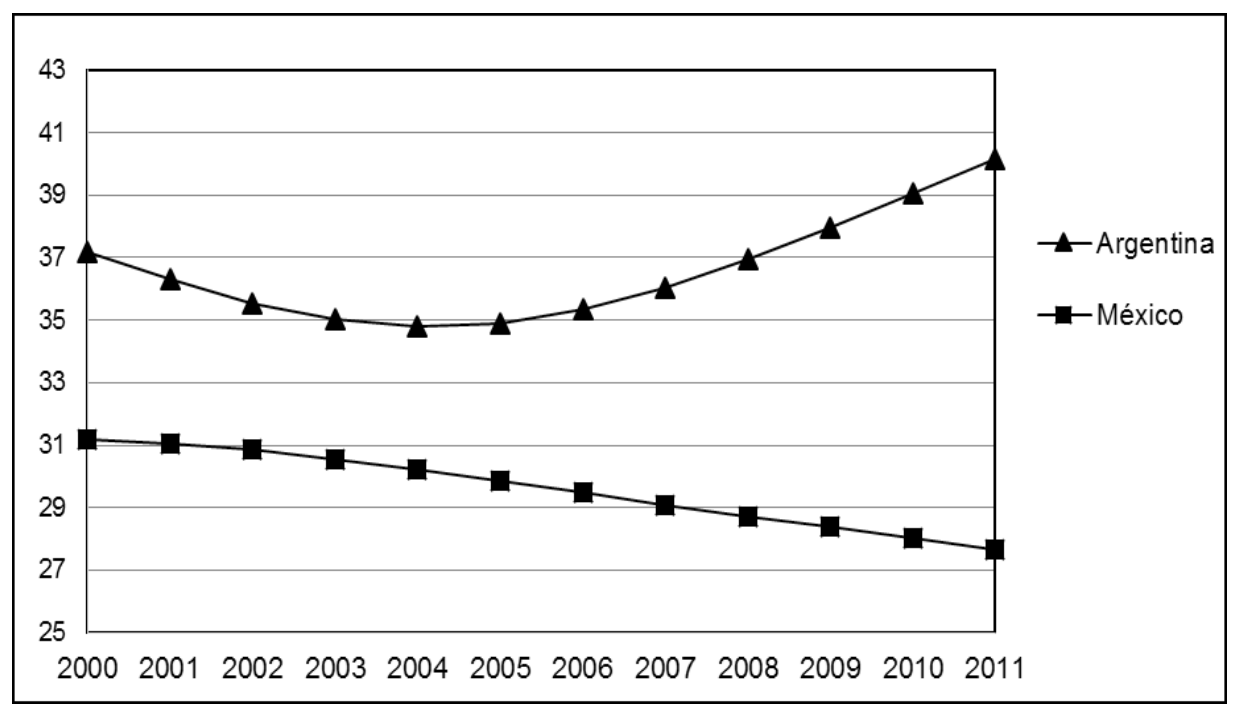

Fuente: elaboración propia a partir de la base de datos de Alarco, Germán, "Participación Salarial y Crecimiento Económico en América Latina, 1950-2011”, Revista CEPAL, núm. 113, 2014, pp. 43-60.

\footnotetext{
${ }^{46}$ OIт, Informe Mundial Sobre Salarios 2014/2015. Salarios y Desigualdad de Ingresos, Ginebra, oІт, 2015, p. 38.

${ }^{47}$ Morlino, Leonardo, La calidad de las democracias en América Latina. Informe para IDEA Internacional, San José, IDEA Internacional, 2014.
} 
Gráfico 2. Índice de Gini en Argentina y México, 2002-2012

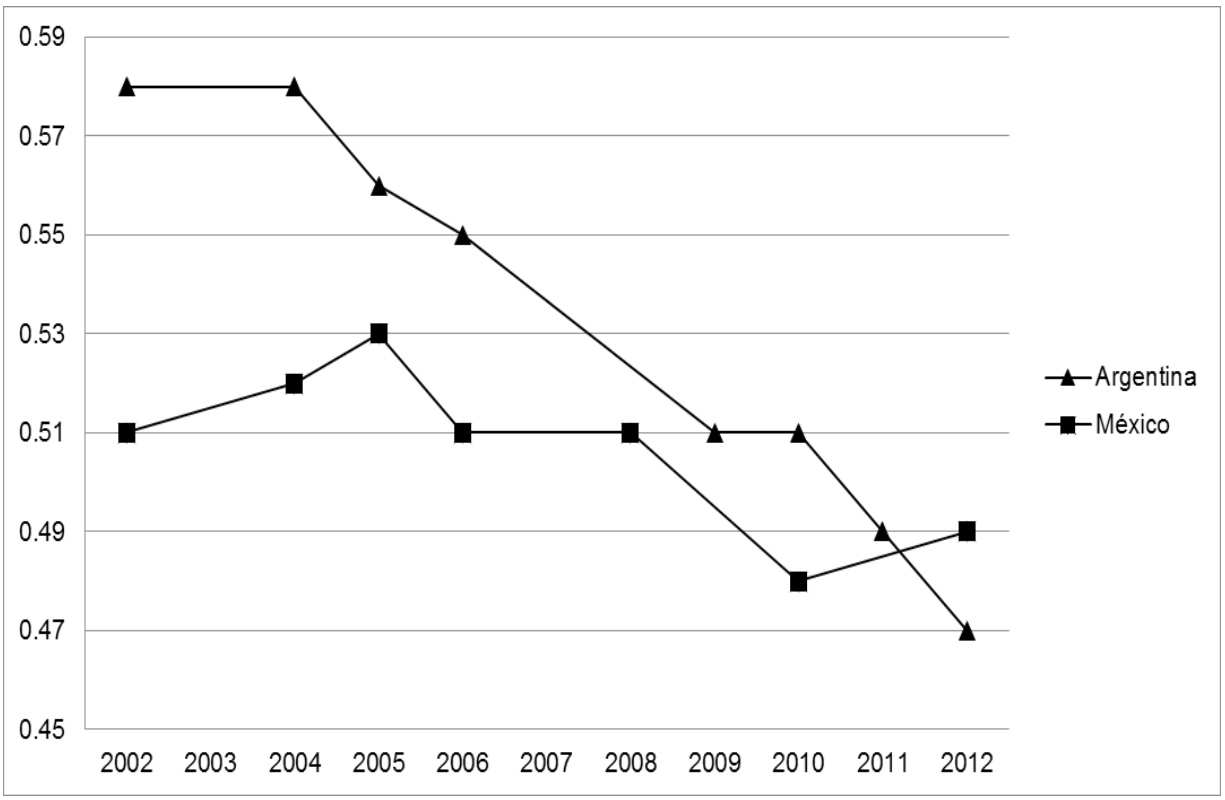

Fuente: Elaboración propia a partir de datos de la CEPAL, disponibles en: http://estadisticas.cepal.org.

En contraste, México ha presentado un escenario sombrío. El ejercicios de los derechos laborales colectivos se ha mantenido seriamente restringido. Como consecuencia, hay fuertes limitaciones para acceder a una ciudadanía plena por parte de la mayoría de la población. La cobertura de la tasa de sindicalización desde 2004 ha venido en constante disminución (se ubicó en 17.5 en 2004, y llegó a 13.6 en 2013). De igual forma la cobertura de la negociación colectiva es muy limitada; en promedio, entre 2007 y 2012 llegó a cubrir 12.32\% de los trabajadores formales La cifra es muy inferior si se considera toda la población ocupada.

Como resultado de ese limitado ejercicio de los derechos laborales colectivos, y por consiguiente un restringido acceso a la voz colectiva (horizontal y vertical) por parte de grandes mayorías ciudadanas, se presenta un gran deterioro de las condiciones socioeconómicas. Por ejemplo, el trabajo informal, entre 2005 y 2015, ha representado en promedio 58.7\% de los ocupados. ${ }^{48} \mathrm{La}$

\footnotetext{
48 INEGI, Encuesta Nacional de Ocupación y Empleo. Cifras en porcentaje del primer trimestre de cada año, para 2005-2015, Ciudad de México, INEGI, 2015.
} 
pobreza, por su parte, entre 2000 y 2014 afectó en promedio a 37\% de la población, incluso con picos que superaron 40\% (en 2000 llegó a 41.1\%; y en el 2014 a 41.2\%). Además, producto de esa imposibilidad de acceder a la voz vertical y horizontal (colectiva e individual), el salario mínimo lleva décadas perdiendo valor, y se ha llegado a posicionar como el segundo más bajo de América Latina. ${ }^{49}$ En consecuencia, en México la participación de los salarios en el PIB ha tendido a ser cada vez menor (véase gráfico 1). Mientras que en el índice de Gini, aun cuando llegó a mostrar mejorías, éstas no han sido sostenidas, e incluso entre 2010 y 2012 se notó un deterioro (véase gráfico 2).

En medio de este panorama, el secretario de Trabajo y Previsión Social, Alfonso Navarrete Prida, en entrevista con el diario La Jornada, ante la pregunta de por qué en México no hay huelgas, señaló, en una suerte de eufemismo, que hay una "transformación de la visión sindical frente a un cambio de paradigma de los gremios”. Además, agregó que no se trata de que los líderes sindicales estén coludidos con los empresarios y el gobierno ("Si en México no estallan los problemas no es porque tengamos dirigentes charros"), ${ }^{50}$ sino de que los trabajadores están satisfechos de las condiciones laborales producto de una buena educación y del sistema de salud ${ }^{51}$ (aunque no indicó cual era la correlación).

En suma, dichas declaraciones reflejan la situación de la representación de los intereses de los trabajadores, y de la continuidad de las limitaciones para acceder al goce de los derechos laborales colectivos en México, lo que ha tenido repercusiones en la calidad de la democracia, tal como lo demuestra la evaluación que hizo Leonardo Morlino para el periodo 1998-2013.52 Lo importante de este estudio es que evaluó e integró en los resultados tres dimensiones centrales: lo referente a los procedimientos electorales; lo referente a los contenidos, incluyendo de esa manera el acceso y efectividad del ejercicio de derechos socio-económicos y de libertades; y lo referente a los resultados, es decir, se avaluó la capacidad gubernamental de satisfacer a la población, implementado políticas que correspondían con sus demandas.

Los resultados del estudio de Morlino mostraron diferencias importantes entre la democracia argentina y la mexicana. Incluso se señala que hay cierta "sorpresa" en los resultados que evidenció Argentina, pues se ubica en una

\footnotetext{
49 oІт, Informe Mundial Sobre Salarios 2014/2015. Salarios y Desigualdad de Ingresos, Ginebra, oІт, 2015.

${ }^{50}$ En México "charro" o "charrismo sindical" hace referencia al líder sindical o sindicato que responde a los intereses de la empresa o gobierno por encima de los intereses de los trabajadores.

${ }^{51}$ Navarrete Prida, Alfonso, "No es mi tarea remover o dejar a los líderes sindicales", La Jornada, miércoles 12 de abril, 2017, p. 12.

52 Morlino, Leonardo, La calidad de las democracias en América Latina. Informe para IDEA Internacional, San José, IDEA Internacional, 2014.
} 
posición elevada, muy cercana a las mejores democracias de la región (Uruguay, Chile y Costa Rica). Es importante destacar que prácticamente en todos los rubros evaluados, Argentina obtuvo un mejor desempeño que México; sin embargo, para efectos de este trabajo, llama la atención lo relativo a libertad, participación política y resposiveness, en tanto son aspectos que se relacionan con la presencia de ciudadanías activas, donde la organización laboral y la existencia de esferas laborales participativas podrían estar jugando un rol determinante.

Aunque es prematuro determinar una correlación en ese sentido para estos casos y en ese periodo específico, podría ser que la tesis demostrada en otros estudios también se cumpla en estos países. ${ }^{53}$ Además, otras mediciones de la calidad de la democracia, como el índice de democracia IEU, dan resultados en el mismo sentido. Para el periodo 2006-2012, Argentina mostró mejorías en su calidad democrática, mientras que México evidenció retrocesos. La tabla 2 muestra los datos del estudio de Morlino.

Tabla 2. Calidad de la democracia en Argentina y México, 1998-2013

\begin{tabular}{|l|c|c|}
\hline & Argentina & México \\
\hline Estado de derecho & 2.27 & 2.37 \\
\hline Rendición de cuentas electoral & 3.75 & 3.47 \\
\hline Rendición de cuentas interinstitucional & 4.34 & 3.25 \\
\hline Participación política & 4.17 & 3.44 \\
\hline Competencia política & 3.93 & 3.68 \\
\hline Libertad & 4.17 & 3.11 \\
\hline Igualdad/Solidaridad & 3.09 & 2.99 \\
\hline Responsiveness & 3.26 & 2.78 \\
\hline Total/a & 3.62 & 3.14 \\
\hline
\end{tabular}

Fuente: Morlino, Leonardo, La calidad de las democracias en América Latina. Informe para ideA Internacional, San José, IDEA Internacional, 2014.

a/ El índice está construido de 0 a 5, siendo 5 la mejor calidad de la democracia posible y de cada aspecto evaluado.

\section{Conclusiones}

La democracia en la región no puede darse por conquistada, sino que está en

\footnotetext{
${ }^{53}$ Abouharb, Rodwan y Cingranell, David, Human Rights and Structural Adjustment, Cambridge, Cambridge University Press, 2007.
} 
constante disputa. Oscila entre periodos de avances y deterioros de su calidad, y el riesgo de retornar al autoritarismo parece estar siempre latente. En un contexto así, el desarrollo de ciudadanías activas, con capacidades para la organización de sus intereses y de la acción colectiva se torna imprescindible.

Los derechos laborales colectivos, como instituciones que dan forma a las relaciones laborales y formas de representación política al margen de los partidos políticos, y que permiten (o bien restringen) la organización de amplios sectores de la población, emergen como recursos significativos para el establecimiento de una ciudadanía que exija y fomente una democracia de calidad. Los derechos laborales colectivos han logrado constituirse como mecanismos para el otorgamiento de voz (horizontal y vertical) a grandes mayorías. No sólo fueron clave en varios procesos de transición hacia la democracia (Polonia, Brasil, Uruguay, Chile, Argentina, entre otros), sino que durante el siglo xxI han demostrado su valía para que se logren avances en la consolidación de democracias de calidad, a través de aspectos sustantivos de estos sistemas de representación política (contenidos y resultados).

\section{Bibliografía}

Alarco, Germán, "Participación Salarial y Crecimiento Económico en América Latina, 1950-2011”, Revista CEPAL, núm. 113.

Abouharb, Rodwan y Cingraneldi, David, Human Rights and Structural Adjustment, Cambridge, Cambridge University Press, 2007.

BARREDA, MAIKEL, "La calidad de la democracia: Un análisis comparado de América Latina”, Política y gobierno, vol. 18, núm. 2.

Bensusán, Graciela, “Organizing Workers in Argentina, Brazil, Chile and Mexico: The Authoritarian-Corporatist Legacy and Old Institutional Designs in a New Context”, Theoretical Inquiries in Law, vol. 17, núm. 1.

Bensusán, Graciela, El modelo mexicano de regulación laboral, México, Flacso, 2000. Bensusán, Graciela y CooK, María Lorena, "State-corporatist Legacies and Divergent Paths”, en Stephen Crowley, María Lorena Cook (eds.), Working through the

Past: Labor and Authoritarian Legacies in Comparative Perspectives, Ithaca, Cornell University Press, 2015.

Bensusán, Graciela, y Cook, María Lorena, "Political Transition and Labor Revitalization in Mexico”, en Daniel Cornfield y Holly McCammon (eds.), Labor Revitalization: Global Perspectives and New Initiatives, Vanderbilt, Elsevier, 2003.

Bensusán, Graciela y Middlebrook, Kevin J., Sindicatos y politica en México: cambios, 
continuidades y contradicciones, Flacso - UAm - Clacso, 2013.

Collier, Ruth Berins y Collier, David, Shaping the Political Arena. Critical Junctures, The Labor Movement, and Regime Dynamics in Latin America, Notre Dame, University of Notre Dame Press, 2002.

Cook, María Lorena, "Labor Reform and Dual Transitions in Brazil and the Southern Core”, Latin American Politics and Society, vol. 44, núm. 1.

Cook, María Lorena, The Politics of Labor Reform in Latin America: Between Flexibility and Rights, University Park, The Pennsylvania State University Press, 2007.

DeAkin, Simon, "Labor Law and Development in the Long Run”, en Shelley Marshall y Colin Fenwick (eds.), Labour Regulation and Development, Cheltenham Geneva, Edward Elgar Publishing - ILo, 2016.

Etchemendy, Sebastián y Collier, Ruth Berins, “Down but not out: Union resurgence and segmented neocorporatism in Argentina (2003-2007)”, Politics \& Society, vol. 35, núm. 3.

FICK, BARBARA, “Not Just Collective Bargaining: The Role of Trade Unions in Creating and Maintaining a Democratic Society", WorkingUSA: The Journal of Labor and Society, vol. 12, núm. 2, 2009.

Francese, Maura y Mulas-Granados, Carlos, "Functional Income Distribution and Its Role in Explaining Inequality”, IMF Working Paper, núm. 15/244, International Monetary Fund, 2015.

Fung, Archon, “Associations and democracy: Between theories, hopes, and realities", Annual review of sociology, vol. 29, núm. 1.

Gordon, Jennifer, "Transnational Labor Citizenship”, Southern California Law Review, vol. 80, 2007.

Hirschman, Albert, Exit, Voice and Loyalty: Responses to Decline in Firms, Organizations and States, Cambridge, Harvard University Press, 1970.

INEGI, Encuesta Nacional de Ocupación y Empleo. Cifras en porcentaje del primer trimestre de cada año, para 2005-2015, Ciudad de México, INEGI, 2015.

Jaumotte, Florence y Osorio Buitrón, Carolina, "Power from the People", Finance \&t Development, vol. 52, núm. 1.

Kolben, Kevin, “Labour Regulation, Human Capacities and Industrial Citizenship”, en Shelley Marshall y Colin Fenwick (eds.), Labour Regulation and Development, Cheltenham - Geneva, Edward Elgar Publishing - ILo, 2016.

Liм, Тімотну C., Doing comparative politics: An introduction to approaches and issues, Boulder, Lynne Rienner Publishers, 2006.

Locke, Richard y Thelen, Kathleen, “Apples and Oranges Revisited: Contextualized Comparison and the Study of Comparative Labor Studies”, Politics \& Society, vol. 23, núm. 3.

Marticorena, Clara, "Revitalización sindical y negociación colectiva en Argentina (2003-2011)”, Perfiles latinoamericanos, vol. 23, núm. 46.

Morlino, Leonardo, La calidad de las democracias en América Latina. Informe para 
IDEA Internacional, San José, IDEA Internacional, 2014.

Navarrete Prida, Alfonso, “No es mi tarea remover o dejar a los líderes sindicales”, La Jornada, miércoles 12 de abril, 2017, p. 12.

Novick, Marta y Tomada, Carlos, “Argentina 2003-2006: Crecimiento Económico con Empleo Decente ¿Un Nuevo Modelo para América Latina?”, en Marta Novick, Carlos Tomada, Mario Damill, Roberto Frenkel y Roxana Maurizio Ginebra (eds.), Tras la crisis: el nuevo rumbo de la politica económica y laboral en Argentina y su impacto, Instituto Internacional de Estudios Laborales, 2007.

O’DonNell, Guillermo, “Sobre las fructíferas convergencias de las obras de Hirschman, salida, voz y lealtad y compromisos cambiantes: reflexiones a partir de la experiencia argentina reciente", en Guillermo 0’Donnell (ed.), Contrapuntos. Ensayos escogidos sobre autoritarismo y democratización, Buenos Aires, Paidós, 1997.

O’Donnell, Guillermo, “Transiciones, Continuidades y Algunas Paradojas”, en Guillermo O’Donnell (ed.), Contrapuntos. Ensayos escogidos sobre autoritarismo y democratización, Buenos Aires, Paidós, 1997.

оाт, Informe Mundial Sobre Salarios 2014/2015. Salarios y Desigualdad de Ingresos, Ginebra, oIT, 2015.

огт, Panorama Laboral 2011: América Latina y el Caribe, Lima, orT, 2011.

oIT, Panorama Laboral 2012. América Latina y el Caribe, Lima, orT, 2012.

Oyserman, Daphna, Coon, Heather \& Kemmelmeier, Markus, "Rethinking individualism and collectivism: evaluation of theoretical assumptions and meta-analyses”, Psychological Bulletin, vol. 128, núm. 1.

Pateman, Carole, Participation and democratic theory, Cambridge, Cambridge University Press, 1970.

PrZEWORSKI, ADAm, “Desigualdad económica, desigualdad política y redistribución”, Conferencia Magistral 40 aniversario de Flacso-México, Ciudad de México, 28 de octubre de 2015.

Przeworski, Adam, "Minimalist Conception of Democracy: A Defense”, en Robert Dahl, Alan Ian Shapiro, y José Antônio Cheibub (eds.), The Democracy SourceBOOK, Massachusetts, The mit Press, 2003.

Przeworski, Adam y Teune, Henry, The Logic of Comparative Social Inquiry, Florida, Robert E. Krieger Publishing Company, 1982.

SÁnchez y SÁnchez, Carlos Luis, "Calidad de la democracia y respuesta política. Responsiveness”, Apuntes Electorales, año 15, núm. 54.

Schmitter, Philippe, "Danger and Dilemmas of Democracy", Journal of Democracy, vol. 5, núm. 2, 1994.

Schwartz, Shalom H., "Individualism-collectivism: Critique and proposed refinements”, Journal of cross-cultural psychology, vol. 21, núm. 2.

SenÉn GonzÁlez, Cecilia, "La revitalización sindical en Argentina durante los 
Kirchner", Revista Trabajo, vol. 5, núm. 8.

Senén González, Cecilia y Del Bono, Andrea, “Introducción”, en Cecilia Senén González y Andrea del Bono, La revitalización sindical en Argentina: alcances y perspectivas, San Justo, Prometeo - Universidad Nacional de la Matanza, 2013.

SenÉn González, Cecilia, Trajtemberg, David y Medwid, Bárbara "La negociación colectiva en la Argentina 1991-2007”, en Cecilia Senén González y Patricia Schettini (comps.), Trabajo y relaciones laborales en Argentina: sector formal e informal, Buenos Aires, Universidad Nacional de La Matanza - Prometeo, 2009.

Stiglitz, Joseph, El precio de la desigualdad, Bogotá, Taurus, 2012. 